\title{
De Novo Nonsense Variant in ASXL3 in a Chinese Girl Causing Bainbridge-Ropers Syndrome: A Case Report and Review of Literature
}

\section{Qin Wang}

Shenzhen Maternity and Child Healthcare Hospital

Jianming Zhang

Shenzhen Maternity and Child Healthcare Hospital

Nan Jiang

Maternity and Children Hospital

Jiansheng Xie

The University of Hong Kong-Shenzhen Hospital

Jingxin Yang

Shenzhen Maternity and Child Healthcare Hospital

Xiaoshan Zhao ( $\nabla$ xszhaoszmch@163.com )

Shenzhen Maternity and Child Healthcare Hospital

\section{Case Report}

Keywords: Bainbridge-Ropers syndrome (BRPS), ASXL3, loss-of-function (LOF), whole exome sequencing (WES), prenatal diagnosis

Posted Date: August 26th, 2021

DOl: https://doi.org/10.21203/rs.3.rs-810120/v1

License: (9) This work is licensed under a Creative Commons Attribution 4.0 International License. Read Full License

Version of Record: A version of this preprint was published at Molecular Genetics \&amp; Genomic Medicine on March 11th, 2022. See the published version at https://doi.org/10.1002/mgg3.1924. 


\section{Abstract}

Background: Bainbridge-Ropers syndrome (BRPS, OMIM \#615485) was first identified in 2013 by Bainbridge et al. and is a neurodevelopment disorder characterized by failure to thrive, facial dysmorphism, and severe developmental delay. BRPS is caused by heterozygous loss-of function (LOF) variants in the additional sex combs-like 3 (ASXL3) gene which are mostly located in two mutational cluster regions (MCR). Due to the limited specific recognizable features and overlapping symptoms with Bohring-Opitz syndrome, clinical diagnosis of BRPS is challenging.

Case presentation: In this study, a 2-year-8-month-old Chinese girl was referred for genetic evaluation of severe developmental delay. Reduced fetal movement was found during antenatal period and bilateral varus deformity of feet was observed at birth. Whole exome sequencing and Sanger sequencing were used to detect and confirm the variant. A novel nonsense variant c.1063G > T (p.E355X) in the ASXL3 gene (NM_030632.3) was identified in the proband and the clinical symptoms were compatible with BRPS. The parents were physical and genetic normal and prenatal diagnosis was requested for her pregnant mother with a negative Sanger sequencing result.

Conclusion: The study revealed a de novo LOF variant in the ASXL3 gene and expanded the mutation spectrum for this clinical condition. By performing a literature review, we analyzed the clinical phenotype with limited fetal features and summarized genetic results of all BPRSs reported so far. More cases study may help to elucidate the function of $A S X L 3$ gene that may be critical to understand the genetic etiology of this syndrome and assist in accurate genetic counselling, informed decision making and prenatal diagnosis.

\section{Background}

Bainbridge-Ropers syndrome (BRPS, OMIM \#615485) is a recently identified neurodevelopmental disorder, characterized by severe developmental delay with feeding problems, hypotonia, ulnar deviation of hands, and characteristic facial changes of prominent forehead, full (everted) lower lip, arched eyebrows, low-set ears, broad nasal tip and anteverted nares [1, 2]. It was first identified in four unrelated probands by Bainbridge et al. in 2013 by using whole-exome sequencing. Frameshift, truncating or splicing variants in the ASXL3 gene have been shown to be responsible for the BRPS. De novo truncating mutations in $A S X L 1$ and $A S X L 2$, which belong to the same gene family as $A S X L 3$, cause Bohring-Opitz syndrome (BOS, OMIM \#612990) and ASXL2-related disorder (OMIM \#612991), respectively, which share clinical manifestations with BRPS [3, 4].

ASXL3 is mammalian homologues of drosophila Additional sex combs-like 3 gene. Together with ASXL 1 and $A S X L 2$, they consist of ASXL family members and are assumed to be epigenetic regulators that are involved in hereditary neurological disorders, malignancies and congenital heart disease (CHD) [4-10]. The ASXL family members share a common domain architecture, namely ASXN and ASXH domains in the N-terminus; ASXM1 and ASXM2 domains in the middle region, and the PHD domain in the C-terminus. 
ASXL3 interacts with BAP1 which functions as a component of the Polycomb repressive deubiquitination (PR-DUB) complex, playing a role in chromatin remodeling on transcriptional regulation[11]. Most pathogenic mutations in $A S X L 3$ locate in $5^{\prime}$ mutation cluster region (MCR) in exon 11 and several variants locate in ASXM2 region in exon 12.

To our knowledge, 54 cases with ASXL3 LOF variants and one splicing mutation have been reported including ten Chinese patients [12-18]. The studies demonstrate clinical heterogeneity exists in the affected subjects and the correlation between the position of variants and the severity of the phenotype is uncertain $[5,17,19]$. Nonsense variants in MCR found in phenotypically normal individuals, germline and postzygotic mosaicism were also reported $[13,17,20]$.

In this study, we present the detection of de novo nonsense variant c.1063G > T (p.E355X) in the ASXL3 gene (NM_030632.3) in a Chinese patient and expanded the clinical phenotypes of BRPS. Prenatal diagnosis was made for her pregnant mother with a negative Sanger sequencing result. The proband's phenotypic features was consistent with BRPS and molecular result expands the genetic spectrum. More cases study may help to elucidate the function of $A S X L 3$ gene that may be critical to understand the genetic etiology of this syndrome and assist in accurate genetic counselling, informed decision making and prenatal diagnosis.

\section{Case Presentation}

\section{Clinical phenotype}

A 2-year-8-month-old Chinese girl was referred for genetic evaluation of developmental and speech delay at the genetic counselling clinic in Shenzhen Maternal and Child Healthcare Hospital. The couple performed genetic counselling for the next pregnancy. Blood samples were collected from the patient and their parents. Detailed clinical information was obtained by the clinicians examining the patient. The present study was approved by the hospital's Institutional Review Board and written informed consent for publication of their clinical details and/or clinical images was obtained from the parents.

The patient was the first child of non-consanguineous Chinese couple with unremarkable family history and her mother was pregnant for the genetic counselling (Fig. 1). Physical examination showed her weight was $10.9 \mathrm{~kg}$ at 10 th percentile, her height was $88 \mathrm{~cm}$ at 10 th percentile and her head circumference was $47 \mathrm{~cm}$ at $10-25$ th percentile. The girl was born at the 39th gestational week by Cesarean section with deceased fetal movement during antenatal period. At birth she was found to have bilateral varus deformity of feet and internal rotation of femur. She was able to hold her head up by 6 months and sat without support at 10 months. She stood by herself at 12 months and walked freehand at 20 months. His feeding problem was also complicated with gastroesophageal reflux at 12 months old. She was able to say baba and mama at 2 years old. The girl had tapering fingers and CT, MRI, hearing test were normal. Sleeping disturbance was observed from infancy. Mild craniofacial features were arched eyebrows, prominent forehead, low-set cupped ears, high-arched palate, hypertelorism with downslanting palpebral 
fissures and everted lower lip. The parents planned to enrolled her to special child care kindergarten for special training.

\section{Genetic Analysis}

We performed whole exome sequencing on proband. The genomic DNA was extracted from peripheral blood. The mean read depth of the protein-coding regions was $56.2 x$ and an average of $92.3 \%$ of coding sequences (CDS) were sequenced by 20 or more reads. We found a pathogenic variant, c. 1063G > T (p.E355X) in the ASXL3 gene (NM_030632.3) with dominant inheritance pattern, causing BainbridgeRopers syndrome (BRPS) and matching the proband clinically. We then sequenced parent's DNA to perform trio analysis. The result confirmed the patient carried de novo dominant nonsense variant and the parents were absent from the variant. The proband's mother had amniocentesis at 20th gestational week and Sanger sequencing result of c.1063G > T was negative (Fig. 1). Primers used to amplify the mutant sequence were ASXL3-1063-F (5'TCTGTGCCTTGTGATTTA3') and ASXL3-1063-R (5'TGCTTTCAGGGTTAGTTC3').

\section{Discussion And Conclusions}

In the present study, whole exome sequencing detected a novel heterozygous nonsense variant c.1063G > T (p.E355X) in the ASXL3 gene (NM_030632.3) in a Chinese girl with BRPS. Sanger sequencing confirmed the wildtype in parents and fetal which revealed the dominant de novo pattern. In silico prediction analysis demonstrated this variant had damaging or disease-causing effects (PVS1 + PM1 + PM2) according to ACMG guidelines [21]. ASXL3 c.1063G > T (p.E355X), locating in mutation cluster region (MCR) in exon 11, is a nonsense variant which causes the truncating of the protein. The proband presented in this study exhibit most of the typical clinical manifestations of BRPS including feeding difficulties, hypotonia, absent speech, intellectual disability and facial dysmorphism. Our study reports a novel variant in $A S X L 3$ which enriches the genetic spectrum and further emphasizes the dominant inheritance of BRPS.

BRPS was first described in 2013, in four patients with ASXL3 de novo mutations. To date, 54 patients with a wide age range from 4 months to 47 years were reported [1, 2, 5, 11-20, 22-30]. Mutation spectrum of $A S X L 3$ and the number of patients were listed in Table 1. Forty-five different pathogenic mutations in ASXL3 were summarized with 22 patients in exon 11 and 32 patients in exon 12. All the described mutations are nonsense or frameshift variants except one splice site mutation carried by two separate patients. In addition to the spice site mutation, variant c.3106C > T (p.Arg1036*) was detected in five patients and c.4330 $>T$ (p.Arg1444*) was detected in three patients, which suggested they are likely mutational hotspots. The prediction that phenotype severity decreases as the variants occur further away from the 5 '-end of exon 11 and towards the 3 '-end needs to be further confirmed[19]. More patients should be collected to study the genotype-phenotype correlation in this severe atypical neurodevelopmental disorder. 
In our study, the proband was diagnosed as BRPS and the parents were phenotypic and genetic normal. Considering the recurrence risk, the pregnant mother still had prenatal diagnosis and the negative result excluded the gonadal mosaicism of the couple. The prenatal diagnosis of BRPS was challenging owing to its limited ultrasonography findings. Polyhydramonios, decreased fetal movements in late pregnancy and anthrogryposis on ultrasound were observed on separate fetus $[5,11,20,29]$. In our study, reduced fetal movement was found during pregnancy and bilateral varus deformity of feet was observed at birth, which were consistent with the reported fetal cases. The limited prenatal cases revealed that reduced fetal movements and anthrogryposis on ultrasound maybe the clinical features of the BRPS fetus. Prenatal imaging results combining with molecular genetic analysis would help a clear diagnosis. In previous studies, three families had sibling recurrent variants and one family had mosaicism variant in the proband. Germline mosaicism in one of the parents seems to be a more likely explanation [20,23]. Within the families, behavioral phenotype diversity and difference in intellectual development among siblings with consistent variants have demonstrated the heterogeneity of this clinical condition [20]. A nonsense $A S X L 3$ mutation carried by a patient with developmental delay and hypotonia was inherited from the clinically unaffected father, which suggests incomplete penetrance [13,31]. Accurate genetic counselling should be carefully performed in families of a child with a dominant genetic condition caused by a de novo pathogenic variant.

De novo truncating ASXL3 mutations are predicted to promote nonsense-mediated decay (NMD) and disrupt the normal activity of the Polycomb repressive deubiquitination (PR-DUB) complex. Transcriptome analysis of $A S X L 3$ fibroblasts from patients with BRPS resulting the differentially expressed genes (DEGs) have suggested that $A S X L 3$ is involved in transcriptional regulation of brain development genes [11]. Besides a causing dominant gene responsible for BRPS phenotype, compound heterozygous variants in $A S X L 3$ have also been proposed to cause congenital heart disease (CHD) and recurrent mutations in $A S X L 3$ have been identified in specific subset of cancer [7-9]. Potentially, the underlying mechanism may be that different kinds of variants in $A S X L 3$ cause various mRNA expression and protein levels across clinical conditions. Compound heterozygous variants in ASXL3 causing BRPS-like features with primary IGF1 deficiency proposed the additively or synergistically effect and the complex interaction network of $A S X L 3$ [32]. To date, there are three loss-of-function mutations (p.R322*, p.S887Ffs 2 , p.P2037Hfs*43) in the ASXL3 gene within the ExAC (Exome Aggregation Consortium) dataset. These mutations are identified in phenotypically normal individuals and may be presumably explained by escaping from nonsense-mediated RNA decay due to their location. In addition, these mutations probably occurred post-zygotically or late in embryogenesis which is supported by some extent that somatic mutations occur in ASXL3 in cancers.

In conclusion, our study reports a novel heterozygous nonsense variant in a Chinese patient which expands the mutation spectrum in ASXL3 and clinical features of BRPS. Insights into the genetic etiology of BRPS are dependent on the causative gene study and further functional research. More case reports may help to elucidate the function of $A S X L 3$ that may be critical to understand the etiology of the disease and facilitate genetic counselling and future prenatal testing. 


\section{Abbreviations}

BRPS: Bainbridge-Ropers syndrome

LOF: loss-of function

ASXL3: additional sex combs-like 3

MCR: mutational cluster regions

WES: whole exome sequencing

BOS: Bohring-Opitz syndrome

CHD: congenital heart disease

PR-DUB: Polycomb repressive deubiquitination

DEGs: differentially expressed genes

ExAC: Exome Aggregation Consortium

\section{Declarations}

- Ethics approval and consent to participate- A statement on ethics approval and consent for this study was approved by the Institutional Ethics Review Board, Shenzhen Maternity and Child Healthcare Hospital. Written informed consent for publication of their clinical details and/or clinical images was obtained from the parents to take part in this study.

- Consent for publication-Written informed consents for publication of their clinical details and/or clinical images were obtained from the patient or parents.

- Availability of data and material - The datasets generated during and/or analyzed during the current study are available from the corresponding author on reasonable request.

- Competing interests- The authors declare that they have no competing financial interests in this study.

- Funding- This work was supported by Shenzhen Science and Technology Innovation Commission (JCYJ20180306173032583). The funder had no role in study design, data collection and analysis, decision to publish, or in the preparation of the manuscript.

- Authors' contributions - WQ, performed exome sequencing, data analysis, literature review and drafted the manuscript; JMZ, NJ and JXY performed Sanger sequencing analysis and patient record management; JSX, performed genetic counselling; XSZ, organized this study, reviewed clinical and laboratory data, and finalized this manuscript. All authors read and approved the final manuscript. 
- Acknowledgements- The authors appreciate the family to taking part in this study and for permission to publish the article.

\section{Corresponding author}

Correspondence to Dr. Xiaoshan Zhao, xszhaoszmch@163.com

\section{References}

1. Bainbridge $M N$, et al. De novo truncating mutations in ASXL3 are associated with a novel clinical phenotype with similarities to Bohring-Opitz syndrome. Genome Med. 2013;5(2):11.

2. Dinwiddie DL, et al. De novo frameshift mutation in ASXL3 in a patient with global developmental delay, microcephaly, and craniofacial anomalies. BMC Med Genomics. 2013;6:32.

3. Hastings R, et al. Bohring-Opitz (Oberklaid-Danks) syndrome: clinical study, review of the literature, and discussion of possible pathogenesis. Eur J Hum Genet. 2011;19(5):513-9.

4. Shashi V, et al. De Novo Truncating Variants in ASXL2 Are Associated with a Unique and Recognizable Clinical Phenotype. Am J Hum Genet. 2016;99(4):991-9.

5. Balasubramanian $\mathrm{M}$, et al. Delineating the phenotypic spectrum of Bainbridge-Ropers syndrome: 12 new patients with de novo, heterozygous, loss-of-function mutations in ASXL3 and review of published literature. J Med Genet. 2017;54(8):537-43.

6. Gelsi-Boyer V, et al. Mutations of polycomb-associated gene ASXL1 in myelodysplastic syndromes and chronic myelomonocytic leukaemia. Br J Haematol. 2009;145(6):788-800.

7. Wei Z, et al. Whole-Exome Sequencing Identifies Novel Recurrent Somatic Mutations in Sporadic Parathyroid Adenomas. Endocrinology. 2018;159(8):3061-8.

8. Fu F, et al., Compound heterozygous mutation of the ASXL3 gene causes autosomal recessive congenital heart disease. Hum Genet. 2020.

9. Micol JB, Abdel-Wahab O, The Role of Additional Sex Combs-Like Proteins in Cancer. Cold Spring Harb Perspect Med. 2016; 6(10).

10. Shukla V, et al. ASXL3 Is a Novel Pluripotency Factor in Human Respiratory Epithelial Cells and a Potential Therapeutic Target in Small Cell Lung Cancer. Cancer Res. 2017;77(22):6267-81.

11. Srivastava A, et al. De novo dominant ASXL3 mutations alter H2A deubiquitination and transcription in Bainbridge-Ropers syndrome. Hum Mol Genet. 2016;25(3):597-608.

12. Zhang $R$, et al. Bainbridge-Ropers syndrome with ASXL3 gene variation in a child and literature review. Zhonghua Er Ke Za Zhi. 2018;56(2):4.

13. Fu C, et al. Chromosomal microarray and whole exome sequencing identify genetic causes of congenital hypothyroidism with extra-thyroidal congenital malformations. Clin Chim Acta. 2019;489:103-8.

14. Qiao L, et al. Novel Nonsense Mutation in ASXL3 causing Bainbridge-Ropers Syndrome. Indian Pediatr. 2019;56(9):3. 
15. Yang L, et al. Bainbridge-ropers syndrome caused by loss-of-function variants in ASXL3: Clinical abnormalities, medical imaging features, and gene variation in infancy of case report. BMC Pediatr. 2020;20(1):287.

16. Lyu Y, Zhang ZD, Gao K, Ma M, Wang J, Gai D, Liu Z. Y., Diagnosis of Bainbridge-Ropers syndrome due to de novo ASXL3 variant by high throughput sequencing. Zhonghua Yi Xue Yi Chuan Xue Za Zhi. 2020;37(4):3.

17. Yu KP, et al. Further expanding the clinical phenotype in Bainbridge-Ropers syndrome and dissecting genotype-phenotype correlation in the ASXL3 mutational cluster regions. Eur J Med Genet. 2021;64(1):104107.

18. Li JR, Lu HZ, Ji Y, Jiang QY, Yang MY. F., Novel mutation in the ASXL3 gene in a Chinese boy with microcephaly and speech impairment: A case report. World J Clin Cases. 2020;8(24):13.

19. Wayhelova $M$, et al. Novel de novo frameshift variant in the ASXL3 gene in a child with microcephaly and global developmental delay. Mol Med Rep. 2019;20(1):505-12.

20. Schirwani S, et al. Mosaicism in ASXL3-related syndrome: Description of five patients from three families. Eur J Med Genet. 2020;63(6):103925.

21. Richards S, et al. Standards and guidelines for the interpretation of sequence variants: a joint consensus recommendation of the American College of Medical Genetics and Genomics and the Association for Molecular Pathology. Genet Med. 2015;17(5):405-24.

22. Verhoeven $W$, et al. Phenotypic characterization of an older adult male with late-onset epilepsy and a novel mutation in ASXL3 shows overlap with the associated Bainbridge-Ropers syndrome. Neuropsychiatr Dis Treat. 2018;14:867-70.

23. Koboldt DC, et al., A de novo nonsense mutation in ASXL3 shared by siblings with Bainbridge-Ropers syndrome. Cold Spring Harb Mol Case Stud. 2018; 4(3).

24. Kuechler A, et al. Bainbridge-Ropers syndrome caused by loss-of-function variants in ASXL3: a recognizable condition. Eur J Hum Genet. 2017;25(2):183-91.

25. Myers KA, et al. Childhood-onset generalized epilepsy in Bainbridge-Ropers syndrome. Epilepsy Res. 2018;140:166-70.

26. Dad R, et al. Hyperventilation-athetosis in ASXL3 deficiency (Bainbridge-Ropers) syndrome. Neurol Genet. 2017;3(5):e189.

27. Chinen $Y$, et al. Mild prominence of the Sylvian fissure in a Bainbridge-Ropers syndrome patient with a novel frameshift variant in ASXL3. Clin Case Rep. 2018;6(2):330-6.

28. Hori I, et al. Novel splicing mutation in the ASXL3 gene causing Bainbridge-Ropers syndrome. Am J Med Genet A. 2016;170(7):1863-7.

29. Bacrot $S$, et al. Whole exome sequencing diagnoses the first fetal case of Bainbridge-Ropers syndrome presenting as pontocerebellar hypoplasia type 1. Birth Defects Res. 2018;110(6):538-42.

30. Gou J, et al. Bainbridge-Ropers syndrome:a case report and literature review. Journal of Clinical Pediatrics. 2019;37(03):212-4. 
31. Ropers HH, Wienker T. Penetrance of pathogenic mutations in haploinsufficient genes for intellectual disability and related disorders. Eur J Med Genet. 2015;58(12):715-8.

32. Giri D, et al., Novel compound heterozygous ASXL3 mutation causing Bainbridge-ropers like syndrome and primary IGF1 deficiency. Int J Pediatr Endocrinol. 2017; 2017: 8.

\section{Table}

Due to technical limitations, table 1 is only available as a download in the Supplemental Files section.

\section{Figures}
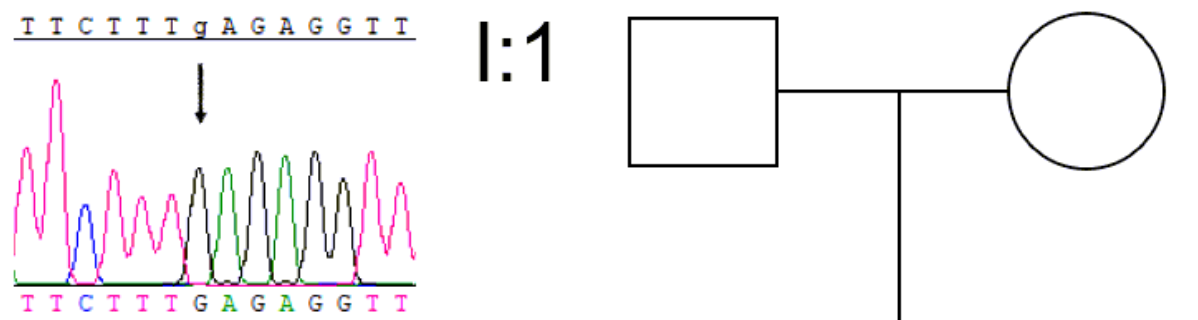

$\mathrm{l}: 2$
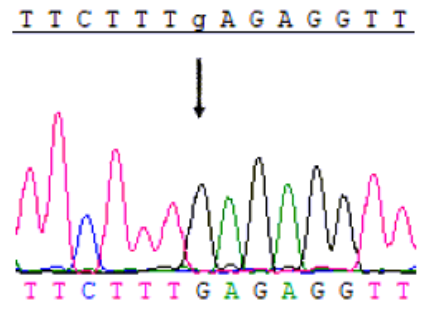

I I C I I I g A G A G G I I
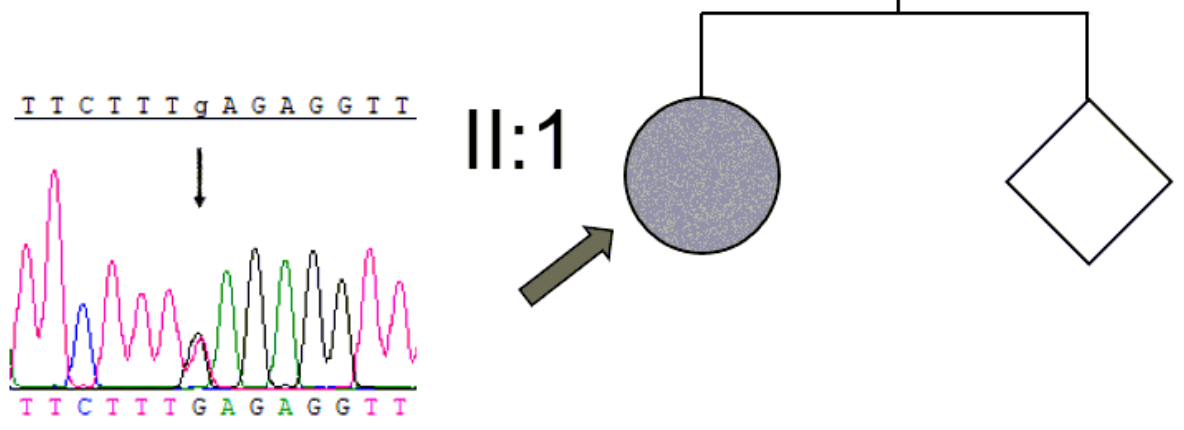

$\mathrm{II}: 2$

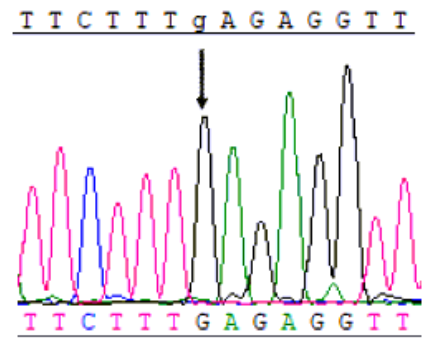

\section{Figure 1}

Pedigree of the proband and Sanger sequencing of family member at position c.1063G $>T$ (p.E355X) in the ASXL3 gene. Family tree of the proband shows the affected patient (Il:1) born from nonconsanguineous parents. Sanger sequencing validates the exome sequencing variant of c.1063G $>T$ (p.E355X) in the ASXL3 gene (NM_030632.3) in the proband (II:1). The father (l:1), mother (I:2) and the fetal (II:2) were variant negative.

\section{Supplementary Files}


This is a list of supplementary files associated with this preprint. Click to download.

- Table1.xlsx 